\title{
Impossibility of VBB Obfuscation with Ideal Constant-Degree Graded Encodings
}

\author{
Rafael Pass* abhi shelat ${ }^{\dagger}$
}

April 24, 2015

\begin{abstract}
A celebrated result by Barak et al (JACM'12) shows the impossibility of general-purpose virtual black-box $(V B B)$ obfuscation in the plain model. A recent work by Canetti, Kalai, and Paneth (TCC'15) extends this result also to the random oracle model (assuming trapdoor permutations).

In contrast, Brakerski-Rothblum (TCC'15) and Barak et al (Euro-

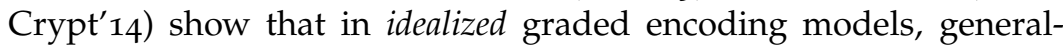
purpose VBB obfuscation indeed is possible; these construction require graded encoding schemes that enable evaluating high-degree (polynomial in the size of the circuit to be obfuscated) polynomials on encodings.

We show a complementary impossibility of general-purpose VBB obfuscation in idealized graded encoding models that enable only evaluation of constant-degree polynomials (assuming trapdoor permutations).
\end{abstract}

\footnotetext{
*Cornell University, rafael@cs.cornell.edu. Work supported in part by a Alfred P. Sloan Fellowship, Microsoft New Faculty Fellowship, NSF Award CNS-1217821, NSF CAREER Award CCF-0746990, NSF Award CCF-1214844, AFOSR YIP Award FA9550-10-10093, and DARPA and AFRL under contract FA8750-11-2-0211. The views and conclusions contained in this document are those of the authors and should not be interpreted as representing the official policies, either expressed or implied, of the Defense Advanced Research Projects Agency or the US Government.

${ }^{+}$U. of Virginia abhi@virginia.edu. Work performed while visiting Cornell Tech, and supported by NSF CAREER Award 0845811, NSF TC Award 1111781, NSF TC Award 0939718, DARPA and AFRL under contract FA8750-11-C-oo80, Microsoft New Faculty Fellowship, SAIC Scholars Research Award, and Google Research Award.
} 


\section{Introduction}

The goal of program obfuscation is to "scramble" a computer program in order to hide its implementation details (making it hard to "reverse-engineer") while preserving its functionality (i.e, input/output behavior). The most desirable notion of security-called virtual black-box security (VBB) $\left[\mathrm{BGI}^{+} \mathrm{OI}\right]$ requires that any bit of information an attacker can learn from the obfuscated code can be simulated using only black-box access to the functionality. ${ }^{1}$ The celebrated result of Barak et al. $\left[\mathrm{BGI}^{+} \mathrm{OI}\right]$, however, demonstrates a strong impossibility result regarding VBB obfuscation: they show the existence of families of functions for which black-box access to $f_{s}$ (for a randomly chosen $s$ ) does not leak any advantage in guessing even a single bit of $s$, but the code of any program that computes $f_{s}$ allows recovery of the entire secret $s$. The idea behind their impossibility result is to consider a function $f_{s}$ that, roughly speaking, satisfies two properties 1 ) the function is not learnable (thus given black-box access to it, it is hard to find a concise representation of it), but 2) on input a program $\Pi$ that computes the function $f_{s}, f_{s}(\Pi)$ reveals some secret. The code of the obfuscated program is thus an input on which the function releases the secret, yet the secret cannot be recovered using just black-box access to the function.

This impossibility result, however, only applies in the plain model in which the obfuscated code is a standard circuit that does not make oracle calls to external functionalities (or else, we cannot feed this code as an input to the function). In contrast, Canetti and Vaikuntanathan [CV13] show an obfuscator for $\mathrm{NC}^{1}$ circuits in an idealized composite-order group with special pseudo-free properties. More recently, Brakerski and Rothblum [BR14a] and Barak, Garg, Kalai, Paneth and Sahai [BGK ${ }^{+}$13], following the breakthrough obfuscation construction of Garg, Gentry, Halevi, Raykova, Sahai, and Waters $\left[\mathrm{GGH}^{+} 13 \mathrm{~b}\right]^{2}$, demonstrate VBB obfuscation for all polynomial-size circuits in the idealized graded encoding [GGH13a] (a.k.a. "approximate" multilinear map [BSo3, Rot13]) model.

In the idealized graded encoding model [BR14a, $\left.\mathrm{BGK}^{+} 13\right]$, players have black-box access to a field $\mathbb{F}_{p}$ (where $p$ is a prime), but they can only perform certain restricted operations on field elements, and determine

${ }^{1}$ A similar simulation-based, but even stronger, notion of security was previously defined by Hada [Hadoo]. Even earlier, Canetti [Can97] considered a similar notion of security (without explicitly referring to obfuscation) for the special case of what is now referred to as point-function obfuscation.

${ }^{2}$ The construction of $\left[\mathrm{GGH}^{+} 13 \mathrm{~b}\right]$ was proved to satisfy the weaker notion of indistinguishability obfuscation in an idealized "matrix-multiplication" model. 
whether an expression evaluates to o. For instance, the simplest form of graded encodings of [GGH13a] enables computing all polynomials of some (a-priori) bounded polynomial degree, and determine whether the polynomial evaluates to 0 ; this is referred to as a "zero-test query". ${ }^{3}$ Note that a generic group [Shog7] model for $\mathbb{Z}_{p}^{*}$ where $p$ is a prime can be viewed as a special-case of an idealized graded encoding model in which operations are restricted to be linear (i.e., degree 1 polynomials). Degree two graded encodings capture idealized groups with bilinear maps.

A natural question is whether weaker idealized models such as the generic group model or idealized groups with bilinear maps suffice for obtaining VBB obfuscation for polynomial-size circuits. This question was first addressed by Lynn, Prabhakaran and Sahai [LPSo4] who showed positive obfuscation results for specific functions in the Random Oracle model [BR93] where all players have oracle access to a truly random function; they left open the question of whether general-purpose obfuscation in the Random Oracle model is possible. This open question was recently answered in an elegant work by Canetti, Kalai and Paneth [CKP15] who show that the impossibility result of $\left[\mathrm{BGI}^{+}{ }^{\mathrm{O}} \mathrm{]}\right.$ ] also extends to the Random Oracle Model. [CKP15] in turn left open the questions of whether generalpurpose VBB obfuscation in more sophisticated idealized models (such as the generic group model) is possible.

Our Results In this work, we show impossibility of VBB obfuscation in idealized graded encoding models that restrict zero-tests to degree- $d$ polynomials, where $d$ is a constant.

Theorem 1 (Informally stated). Assume the existence of trapdoor permutations. Then there exists a family of functions $F$ such that there do not exists a $V B B$ obfuscator for F in idealized degree-d graded encoding models, where $d$ is a constant.

Our theorem stands in contrast with the results of [BR14a] and $\left[\mathrm{BGK}^{+}{ }_{13}\right]$ which indeed show feasibility of general-purpose VBB obfuscation in an idealized graded encoding model that allows for high-degree (polynomial in the size of the circuit being obfuscated) zero-test queries.

The obfusctor construction of $\left[\mathrm{BGK}^{+}{ }_{13}\right]$ actually satisfies subexponential VBB security (that is security holds also with respect to subexponentialsize attackers). We finally remark that our main theorem extends to rule

${ }^{3}$ The constructions in $\left[\mathrm{BR}_{14} \mathrm{a}, \mathrm{BGK}^{+}{ }_{13}\right]$ require certain additional "set-based" restrictions on polynomials; we return to this in Section 2.2. 
out general-purpose VBB obfuscation with subexponential security also in idealized graded encoding models that allow for $n^{\alpha}$-degree (where $\alpha<1$ and $n$ is the description length of the function being obfuscated) zero-test queries.

\section{Definitions and Preliminaries}

\subsection{Virtual Black-box Obfuscation}

We recall the definition of approximate VBB obfuscation from Barak et al $\left[\mathrm{BGI}^{+}{ }^{\mathrm{O}}\right.$ ], and Canetti, Kalai, and Paneth [CKP15], but generalize it for any family of oracles $M$ that are indexed by a security parameter.

Definition 1 ( $\epsilon$-Approximate VBB Obfuscation in an Oracle model [CKP15, BGK $\left.\left.^{+}{ }_{14}\right]\right)$. For a function $\epsilon: \mathbb{N} \rightarrow\{0,1\}$, an obfuscator $\mathcal{O}$ is a secure $\epsilon$ approximate vitrual black-box (VBB) obfuscation for the family $F$ in the M-oracle model if it satisfies the following properties:

- Approximate Functionality: for all $n \in \mathbb{N}, k \in\{0,1\}^{n}$ :

$$
\operatorname{Pr}\left[\mathcal{O}^{M_{|k|}}(k)(x) \neq F_{k}(x)\right] \leq \epsilon(n)
$$

where the probability is over the choice of $x$ and the coins of $M$ and $\mathcal{O}$.

- Virtual Black-Box (VBB): for every poly-size adversary $A$, there exists a poly-size simulator $S$ and a negligible function $\mu$ such that for every $k \in$ $\{0,1\}^{*}$ :

$$
\left|\operatorname{Pr}\left[A^{M_{|k|}}\left(\mathcal{O}^{M_{|k|}}(k)\right)=1\right]-\operatorname{Pr}\left[S^{F_{k}}\left(1^{|k|}\right)=1\right]\right| \leq \mu(|k|)
$$

where the probabilities are over the coins of $M, \mathcal{O}$, adversary $A$ and the simulator $S$.

We simply say that $\mathcal{O}$ is a secure $V B B$ obfuscator if $\epsilon=1$. We further say that $\mathcal{O}$ is a secure (e-approximate) obfuscation in the plain model for the family $F$ if it is a secure ( $\epsilon$-approximate) obfuscation for the family $F$ in the $\perp$-oracle model where the $\perp$-oracle returns $\perp$ on every query.

We finally say that $\mathcal{O}$ is subexponentially-secure if the VBB condition holds with respect to any subexponential-size $A$ and a subexponential-size $S$.

4That is, whose circuit size is bounded by $T(n)=\operatorname{poly}\left(2^{n^{\alpha}}\right)$ for any $0<\alpha<1$. 
Let us remark that our definition of subexponentially-secure VBB obfuscation is incomparable to the definition of VBB obfusaction: it is stronger in that we require simulation also of subexponential-size attackers; it is weaker in that we allow the simulator to be subexponential size (even if the attacker only is polynomial size).

We later use the following theorem by Bitansky and Paneth [BP13].

Theorem 2 ([BP13]). Assuming the existence of trapdoor permutations, there exists a family of polynomial-time computable functions $F$ such that a polynomialsize 0.8-approximate VBB obfuscator for F does not exist.

The following extension of their theorem follows by relying on stronger trapdoor permutations.

Theorem 3 (scaled-up version of [BP13]). Assuming the existence of subexponentiallysecure 5 trapdoor permutations, there exists a family of polynomial-time computable functions F such that a subexponential-size 0.8-approximate subexponentiallysecure VBB obfuscator for $F$ does not exist.

\subsection{Idealized Graded Encodings}

We now define the ideal level- $d$ graded encoding oracle. For simplicity of notation, we consider an oracle that has the size of the field hard-coded. Our model, inspired by the formalism from [PST13, BR14a, BGK ${ }_{14}$, Sho97], considers a simple idealized graded encoding oracle which enables players to a) encode an element $v$ under a "label" $l$, and receive a random "handle" $h$ in return, and b) to make "legal" zero-test queries on these encodings: a zero-test query is a formal polynomial $p$ on variables $\vec{h}$, which evaluates to true iff $p(\vec{v})=0$, where for every $i, v_{i}$ is the value encoded under handle $h_{i}$. The legality of a query is determined by a legality-predicate $g: g(p, \vec{l})$ outputs $\mathbf{I}$ if the query is deemed legal, where $\vec{l}$ are the labels corresponding to the handles $\vec{h}$. In this work we consider a natural class of "well-formed" legality predicates, which, as we shall discuss shortly, generalize all previously used notions of legality.

Definition 2 (Well-formed legality predicate). Given a set of multi-sets (legal label sets) $S$ define the predicate $g_{S}(p, \vec{l})=1$ iff for every monomial $x_{j_{1}} x_{j_{1}} \cdots x_{j_{d}}$ of $p$, it holds that the multi-set $\left\{l_{j_{1}}, \ldots, l_{j_{d}}\right\} \in S$. We say that a legality predicate $g$ is well-formed if there exists a set $S$ such that $g=g_{S}$.

5 That is, security holds against all circuits of size is bounded by $T(n)=\operatorname{poly}\left(2^{n^{\alpha}}\right)$ for any $0<\alpha<1$. 
For instance, to capture:

- idealized groups [Shog7] (where we do not allow any multiplications), consider the predicate $g_{S}$ corresponding to the set $S=\{1\}$ (and requiring that all encodings are made under the label 1 ). If we want to capture groups with bilinear maps, $g(p, \vec{l})$ outputs 1 iff $p$ has degree higher than 2.

- "simple" $d$-level graded encodings of [GGHiza], consider the predicate $g_{S}$ corresponding to the set $S$ where $\left\{l_{j_{1}}, \ldots, l_{j_{m}}\right\} \in S$ iff $\sum_{i \in[m]} l_{j_{i}}=$ $d$ (and requiring that all encodings are made under a label $l \in[d]$ that represents the element's "level").

- "set-based" $d$-level graded encodings [GGH13a, BR14b, BGK ${ }^{+} 14$ ], consider the predicate $g_{S}$ corresponding to the set $S$ where $\left\{l_{j_{1}}, \ldots, l_{j_{m}}\right\} \in$ $S$ iff the disjoint union of labels $l_{j_{i}}$ where $i \in[m]$ is the set $\{1,2, \ldots, d\}$, i.e. $\sqcup_{i \in[d]} l_{j_{i}}=[d]$ (and requiring that all encodings are made under a label $l$ that is a subset of $[d]$ ).

Additionally, to capture secret-key encodings in which only the obfuscator can create new encodings, we follow $\left[\mathrm{BGK}^{+}{ }_{14}\right]$ and require that encodings can only occur once upon initialization; after initialization no more encodings can be performed.

We can now formally define the ideal graded encoding oracle.

Definition 3 (Ideal graded encoding oracle). The oracle $M_{q}^{g}=$ (enc, zero) is a stateful oracle, parameterized by integer $q$ and a legality predicate $g$, that responds to queries in the following manner:

1. Upon initialization (and only then): the activator may adaptively make any number of queries of the form enc $(v, l)$; for each such query $M_{q}^{g}$ picks a uniformly random "handle" $h \in\{0,1\}|q|$, stores the tuple $(v, l, h)$ in a list $\mathcal{L}_{O}$ and returns $h$.

2. On input a query zero $(p)$ where $p$ is a formal polynomial over variables $h_{1}, \ldots, h_{m}$, each of which is represented as a string of length $3|q|$ (corresponding to some handle), $M_{q}^{g}$ does the following:

(a) For each $i \in[m]$, retrieve a tuple $\left(v_{i}, l_{i}, h_{i}\right)$ from the state $\mathcal{L}_{O}$; if no such tuple exists, it returns false.

(b) (Illegal query) If all tuples are retrieved, return false if $g(p, \vec{l}) \neq 1$ 
(c) (Zero test) Finally, return true iff $p\left(v_{1}, \ldots, v_{n}\right)=0 \bmod q$, and false otherwise.

We say that $M$ is an ideal graded encoding oracle if $M=\left\{M_{q_{1}}^{g_{1}}, M_{q_{2}}^{g_{2}}, \ldots\right\}$, and for every $n \in \mathbb{N}, q_{n}$ is a prime, $\left|q_{n}\right|>n$ and $g_{n}$ is a well-formed legality predicate. Finally, we say that $M$ is a degree-d $(\cdot)$ ideal graded encoding oracle if for all $n \in \mathbb{N}, g_{n}(p, \vec{l})$ returns 0 when $\operatorname{deg}(p)>d(n)$.

A Remark on the Model: We remark that, following [PST13], for simplicity of notation, we do not directly allow players to create new encodings by adding and multiplying old ones (as in the definitions of [BR14a, $\left.\left.\mathrm{BGK}^{+}{ }_{14}\right]\right)$. This restriction is without loss of generality since a) an obfuscator "knows" all values it has previously encoded (since it needs to explicitly provide them to the encoding oracle) so instead of operating on old encodings, it can simply operate on the actual values and simply create a new encoding of the resulting value ${ }^{6}$, and $b$ ) when evaluating the obfuscated code, operations on encodings can be simulated by "bogus" (random) handles, and emulating zero-test queries by appropriately modifying the zero-test polynomial $p$ to take into account the previously performed operations.

Feasibility of VBB obfuscation in idealized graded encoding models We note that the result of $\left[\mathrm{BR}_{14} \mathrm{~b}, \mathrm{BGK}^{+}{ }_{14}\right]$ demonstrate feasibility of VBB obfuscation in idealized ("set-based", as described above) graded encoding models that allow zero-test queries with super-constant degree.

Theorem 4 ( [BR14b, BGK $\left.\left.{ }^{+}{ }_{14}\right]\right)$. Under the LWE assumption ${ }^{7}$, for every polynomial $p(\cdot)$, there exists a (polynomial-time computable) sequence of well-formed legality predicates $g_{1}, g_{2}, \ldots$, such that for any ideal graded encoding oracle $M=$ $\left\{M_{q_{1}}^{g_{1}}, M_{q_{2}}^{g_{2}}, \ldots\right\}$, there exists a polynomial-size obfuscator $O^{8}$ such that $O$ is a 0 approximate VBB obfuscation for the class of $p(\cdot)$-sized circuits in the $M$ model.

We furthermore note that their construction also satisfies subexponential VBB security (assuming an appropriate subexponential strengthening of LWE).

\footnotetext{
${ }^{6}$ To make this argument it is important that we allow adaptive encodings during the initialization phase, as opposed to a single non-adaptive encoding query as in the definition of $\left[\mathrm{BGK}^{+}{ }_{14}\right]$.

${ }^{7}\left[\mathrm{BGK}^{+}{ }_{14}\right]$ present unconditionally secure obfuscators for NC1; the LWE assumption is needed to bootstrap up to polynomial-size circuits.

${ }^{8}$ The only non-uniform advice needed is the prime $q_{n}$.
} 


\section{Impossibility of VBB Obfuscation}

Our main theorem is the following.

Theorem 5. Assuming the existence of trapdoor permutations, there exists a family of functions $F$ such that for every constant $d$ and every degree-d ideal graded encoding oracle $M$, a polynomial-size 0.9-approximate VBB obfuscator for $F$ does not exist in the $M$ oracle model.

We briefly review the approach of [CKP15] as we will follow the same high-level structure. [CKP 15$]$ first show that any VBB obfuscator in the Random Oracle model can be transformed into an approximate VBB obfuscator in the plain. They next rely on Theorem 2 to conclude their impossibility result. The first step is achieved by running the original VBB obfuscator in the Random Oracle model by simulating all random oracle queries (with truly random answers). Additionally, to ensure consistency between answers to queries in the obfuscation phase (that cannot be revealed or else security may no longer hold) and answers in the execution of the obfuscated code, [CKP15] add a learning phase to the obfuscator in which most heavy oracle queries (i.e. oracle queries that are made with high probability when running the obfuscated code on random inputs) are discovered; the answers to the heavy queries are hard-coded into the obfuscated code. This ensures that when the obfuscated code is run on a random input, except with inverse polynomial probability (proportional to the number of random inputs used in the learning phase), the obfuscated code will not make any random oracles queries that were not made during the learning phase (i.e. that are not hard-coded), and as a consequence, the obfuscated code correctly computes the function (with high probability). Furthermore, the only difference between the the new (plainmodel) obfuscator and the original (random-oracle-model) obfuscator is that the former leaks the set of heavy queries; since this leak is something that can be learned just by running the obfuscated code of the randomoracle-model obfuscator, VBB security ensures that the same heavy set can be simulated using only black-box access to the function.

As mentioned, we follow the same high-level approach. Our main result (Lemma 6 below) shows how to transform any VBB obfuscator in the constant-degree graded encoding model into an approximate VBB obfuscator in the plain. The proof of Theorem 5 is then concluded by applying Theorem 2. Just as [CKP15], we will run the original (graded-encodingmodel) obfuscator and simulate its oracle queries. But it no longer suffices to simply learn all the heavy queries: the obfuscated code may only ask 
"light" queries (i.e., each query has negligible probability) yet the answer to those queries are correlated (in fact, even determined by) the queries made during the obfuscation phase. For instance, assume that the obfuscator encodes two elements $v_{1}$ and $v_{2}$, and later the obfuscator makes a zero-test query of the form $p\left(v_{1}, v_{2}\right)=a v_{1}+b v_{2}$ where $a$ and $b$ are chosen from some distribution with min-high entropy.

Rather, we show that by running the obfuscated code on sufficiently many random inputs and honestly emulating answers to oracle queries, we can recover a set of linearly independent polynomials in the values $v_{1}, \ldots, v_{\ell}$ encoded during obfuscation phase such that, except with inverse polynomial probability, when the obfuscated code is run on a random input, every zero-test query can be correctly emulated by simply determining whether the zero-test polynomial is a linear combination of polynomials in the stored set. Roughly speaking, the idea is that since we restrict to constant-degree $d$ polynomials, there can be at most $(\ell+1)^{d}$ monomials in the values $v_{1}, \ldots, v_{\ell}$, and thus at most $(\ell+1)^{d}$ linearly independent polynomials in those values. If we record all zero-test polynomials that evaluate to o, then after sufficiently many samples, we have either recovered the full basis (which allows one to correctly answer all remaining zero-test queries), or it is unlikely that a new sample will add another linearly independent polynomial, which in turn means that when the obfuscated code is run on a random input, our emulation only fails with small probability. We finally observe that, just as in [CKP15], leaking the set of linearly independent polynomials does not challenge VBB security as this set (just as the set of heavy random oracle queries in the case of [CKP15]) can be learned from just observing the obfuscated code and can thus be simulated.

We now turn to state and formally prove our main lemma, which combined with Theorem 2 directly concludes our main result (i.e, Theorem 5).

Lemma 6. For every constant $d$ and every degree-d ideal graded encoding oracle $M$, if a family of functions $F$ has a polynomial-size $\epsilon(k)$-approximate $V B B$ obfuscator in the $M$ oracle model, then there exists a polynomial-size $(\epsilon(n)+1 / n)$ approximate 9 VBB obfuscator for $F$ in the plain model.

Proof. Let $M=\left\{M_{q_{1}}^{g_{1}}, M_{q_{2}}^{g_{2}}, \ldots\right\}$ be a degree- $d$ ideal graded encoding oracle for some constant $d$. Let $\mathcal{O}$ be an $\epsilon$-approximate obfuscator for family $F$ in the $M$ oracle model that requests encodings of at most $\ell(|k|)$ elements

$91 / n$ can be replaced by any inverse polynomial by appropriately adjusting the parameters in our proof. 
where $k$ is the index for family $F$; we assume without loss of generality that $\ell(n) \geq 1$. We construct a (non-uniform ${ }^{10}$ ) polynomial-size $(\epsilon(n)+1 / n)$ approximate VBB obfuscator $\mathcal{O}^{\prime}$ for $F$ in the plain model below.

\section{New obfuscator $\mathcal{O}^{\prime}(k)$ :}

1. On input $k$, run $\mathcal{O}(k)$ and simulate the queries to $M_{q_{|k|}}^{g_{k}}$ (i.e., answer the initial enc queries by creating a list $\mathcal{L}_{O}$ of encoded elements as in the definition of $M_{q_{|k|}}^{g k}$, and answer zero $(p)$ queries by evaluating the polynomial $p$ on the "decoded" elements) to compute the obfuscated program $C_{k}$.

2. If $\mathcal{O}(k)$ did not make any initial encoding queries, simply modify the code of $C_{k}$ to honestly emulate the $M$ oracle with some hardcoded uniformly chosen randomness (to generate handles), output this modify code, and halt.

3. Otherwise, set $\mathcal{L}_{c}$ to empty.

4. Repeat until there have been $L=(\ell(|k|)+1)^{d}|k|$ iterations without any new additions to $\mathcal{L}_{c}$ :

(a) Sample random input $x^{j}$.

(b) Run $C_{k}\left(x^{j}\right)$ while simulating zero-test queries to $M$ using the list of encoded elements $\mathcal{L}_{O}$ from Step 1 .

(c) Additionally, whenever a zero-test query zero $(p)$ evaluates to true, record the formal polynomial $p$ if it is linearly independent with all previously stored polynomials in $\mathcal{L}_{c}$. Testing whether $p$ is a linear combination of polynomials in $\mathcal{L}_{c}$ can be performed efficiently through Gaussian elimination (by viewing each monomial as a separate variable).

5. Output a new circuit $C_{k}^{\prime}$ that does the following:

(a) On input $y$, run $C_{k}(y)$.

(b) If $C_{k}(y)$ makes a zero $(p)$ query to $M$, answer true if $p$ is a linear combination of the polynomials in $\mathcal{L}_{c}$ and otherwise answer false.

\footnotetext{
${ }^{10}$ The non-uniformity in our construction is to encode the sequence of primes $q_{1}, q_{2}, \ldots$ that is implicit in the oracle $M^{g}$. If we model the oracle $M$ with a uniform algorithm that picks the field for each security parameter, then our construction below can also be uniform.
} 
Claim 7. Obfuscator $\mathcal{O}^{\prime}$ runs in (non-uniform) polynomial time.

Proof. Recall that $\ell(|k|)$ is an upper bound on the number encodings. As a consequence, there are at most $(\ell(|k|)+1)^{d}$ degree- $d$ monomials in the encodings; thus, there can be at most $(\ell(|k|)+1)^{d}$ linearly independent zero-test polynomials. Since $\mathcal{O}$ continues iterating until there have been $L$ consecutive iterations with no new additions to $\mathcal{L}_{c}$, it follows that there can be at most $L \cdot(\ell(|k|)+1)^{d}$ iterations, each of which can be implemented in polynomial time.

Proposition 1. The obfuscator $\mathcal{O}^{\prime}$ is $(\epsilon(n)+1 / n)$-approximately correct.

Proof. To prove the claim, consider a hybrid obfuscator $\tilde{\mathcal{O}}^{\prime}$ that proceeds just as $\mathcal{O}^{\prime}$ except that it always outputs a program $\tilde{C}^{\prime}{ }_{k}$ that honestly simulates the $M^{g}$ oracle using the state $\mathcal{L}_{O}$ from Stage 1 .

Let $\operatorname{Exp}_{k}$ denote the experiment that consists of running $C_{k} \leftarrow \mathcal{O}(k)$, picking a uniformly random input $x^{*} \leftarrow\{0,1\}^{|k|}$, and outputting 1 iff $C_{k}\left(x^{*}\right)=F_{k}\left(x^{*}\right)$ (and o otherwise). Define $\operatorname{Exp}_{k}^{\prime}$ and $\widetilde{\operatorname{Exp}^{\prime}}{ }_{k}$ in exactly the same way but using $\mathcal{O}^{\prime}$ and $\tilde{\mathcal{O}}^{\prime}$ respectively.

Since $\mathcal{O}$ is $\epsilon(n)$-approximately correct, for every $k \in\{0,1\}^{n}$, we have

$$
\operatorname{Pr}\left[\operatorname{Exp}_{k}=0\right] \leq \epsilon(n)
$$

We also observe that by construction, for every $k \in\{0,1\}^{n}$,

$$
\operatorname{Pr}\left[\operatorname{Exp}_{k}=0\right]=\operatorname{Pr}\left[\widetilde{\operatorname{Exp}^{\prime}}{ }_{k}=0\right]
$$

This directly follows from the observation that the only difference between these experiments is that in $\widetilde{\text { Exp }}$, the obfuscator hard-codes the randomness of $M^{g}$ (needed to generate handles) in the obfuscated code in the event that $\mathcal{O}$ did not make any initial encoding queries. But since in the experiment we only evaluate the obfuscated code on a single input, the outputs of the experiments are identically distributed.

Our goal is now to prove that for $k \in\{0,1\}^{n}$,

$$
\operatorname{Pr}\left[\operatorname{Exp}_{k}^{\prime}=0\right] \leq \operatorname{Pr}\left[\widetilde{\operatorname{Exp}_{k}^{\prime}}=0\right]+1 / n
$$

which concludes the proof of the proposition.

Note that there is only one difference between the program $\tilde{C}^{\prime}{ }_{k}$ produced by $\tilde{\mathcal{O}}^{\prime}$ and the program $C_{k}^{\prime}$ produced by $\mathcal{O}^{\prime}$ when run on the input $x^{*}$ in the above experiments: 
- $C_{k}^{\prime}\left(x^{*}\right)$ may make a zero-test query $\operatorname{zero}(p, \vec{h})$ that should evaluate to true, but $p$ is not in the span of $\mathcal{L}_{c}$ (and thus $C_{k}^{\prime}$ emulates the answer as false, whereas $\tilde{C}_{k}$ honestly emulates the answer as true.) Let $\mathrm{bad}^{i}$ denote the event that this happens for the first time when $\left|\mathcal{L}_{c}\right|=i$.

Let us note that $C_{k}^{\prime}\left(x^{*}\right)$ can never err in the "other direction"; that is, it never answers a zero-test query as true when the answer in fact should be false. This follows from the fact that if $p$ is in the span of $\mathcal{L}_{c}$, then a) all input handles to $p$ correspond to some encoding, and $p$ necessarily evaluates to zero given the encoded value corresponding to those handles, and $b$ ) by the wellformedness condition of $g, g(p, \vec{l})$ necessarily evaluates to true (as $p$ cannot use any monomials not already in use by the polynomials in $\mathcal{L}_{c}$ ).

It follows by construction that conditioned on $\mathrm{bad}^{i}$ not happening for any $i$, experiments $\operatorname{Exp}_{k}^{\prime}$ and $\widetilde{\operatorname{Exp}^{\prime}}{ }_{k}$ proceed identically.

The proof is concluded by the following two claims which show that the probability of any bad event is small. In the following we focus on experiment $\operatorname{Exp}^{\prime}$ but the same arguments straighforwardly hold for $\widetilde{\operatorname{Exp}^{\prime}}$.

Claim 8. For every $i, \operatorname{Pr}\left[\right.$ bad $\left.^{i}\right] \leq 1 / L$.

Proof. For every bad random tape for the experiment on which the event bad $^{i}$ happens, we identify at least $L$ unique good random tapes obtained by swapping the final run on input $x^{*}$ with one of the (at least $L$ ) sampled iterations (using $x_{i}$ ); furthermore, we show that any two distinct bad executions lead to disjoint sets of good executions. We conclude the claim based on the fact that the fraction of bad tapes is at most $1 / L$ and each random tape is equally likely.

Let us now formally specify the mapping $\Phi$ from bad tapes to good tapes, and specify an inverse mapping $\Phi^{-1}$ that given a good tape in the range of $\Phi$ recovers the bad tape it was generated from. The existence of such an inverse map shows that any two distinct bad tapes lead to distinct sets of good tapes, as desired.

Recall that by the proof of Claim $7, m=L \cdot(\ell(n)+1)^{d}$ is a bound on the number of iterations in step 3 . We define a random tape for the experiment $\operatorname{Exp}_{k}$ as $\left(\rho, x_{1}, \ldots, x_{m}, x^{*}\right)$ where $\left(x_{1}, \ldots, x_{m}\right)$ are the inputs sampled to be used in step (3a) of $\mathcal{O}^{\prime}$ (note that not all of those samples may be used), $x^{*}$ is the final input chosen in the experiment, and $\rho$ is the remaining randomness (i.e., the randomness of underlying $\mathcal{O}$ and randomness of $\mathcal{O}^{\prime}$ 
in the event that $\mathcal{O}$ did not make the initial encoding queries). Let $q(R)$ denote the number of samples made in step 3 given the random tape $R$; by construction $L \leq q(R) \leq m$.

We say that a random tape $R=\left(\rho, x_{1}, \ldots, x_{m}, x^{*}\right)$ is bad if $\operatorname{Exp}_{k}^{\prime}(R)$ induces event badi ${ }^{i}$; that is, a) in the evaluations of $C_{k}^{\prime}\left(x_{i}\right)$ for $i \in[q(R)-$ $L, \ldots, q(R)]$, there are no linearly independent zero-test polynomials that evaluate to $\mathrm{o}, \mathrm{b})$ the evaluation of $C_{k}^{\prime}\left(x^{*}\right)$ leads to such a linearly independent polynomial that evaluates to o, and c) the size of $\mathcal{L}_{c}$ is $i$.

Define the mapping $\Phi(R)$ as the set of $L$ random tapes $\Phi(R)=\left\{R_{j}\right\}_{j \in[L]}$ where $R_{j}$ is constructed by swapping the $t^{\text {th }}$ random sample $x_{t}$, where $t=(q(R)-j+1)$, with the last sample $x^{*}$ as follows:

$$
R_{j}=\left(\rho, x_{1}, \ldots, x_{t-1}, x^{*}, x_{t+1}, \ldots, x_{m}, x_{j}\right)
$$

Note that $\operatorname{Exp}_{k}\left(R_{j}\right)$ will not induce event bad ${ }^{i}$ since at least $i+1$ linearly independent polynomials that evaluate to o have been found.

Finally, let $\Phi^{-1}(\cdot)$ be an inverse map that on input a tape $R$, swaps the last sample in the tape with the first sample $x_{t}$ that leads to $i+1$ linearly independent polynomials in the set $\mathcal{L}_{c}$ (and if no such $x_{t}$ exists simply outputs $R$ ). It follows directly by construction that for every bad $R, \Phi^{-1}(\Phi(R))=R$. (Note that in our definition of the inverse map, we make use of the fact that the event bad ${ }^{i}$ is parameterized by $i$.)

By a union bound, it follows from Claim 8 that,

$$
\operatorname{Pr}\left[\exists i \text { s.t. } \text { bad }^{i}\right]=\operatorname{Pr}\left[\operatorname{bad}^{1} \vee \cdots \vee \text { bad }^{\ell^{\prime}(n)^{d}}\right] \leq \frac{\ell^{\prime}(n)^{d}}{L}=\frac{\ell^{\prime}(n)^{d}}{\ell^{\prime}(n)^{d} n}=1 / n
$$

where $\ell^{\prime}(n)=\ell(n)+1$ since as noted in the proof of Claim 7 , the maximum size of $\mathcal{L}_{c}$ is $\ell^{\prime}(n)^{d}=(\ell(n)+1)^{d}$. This concludes that $\mathcal{O}^{\prime}$ is $\epsilon(n)+$ $1 / n$ approximately correct.

Proposition 2. Obfuscator $\mathcal{O}^{\prime}$ satisfies the virtual-black box property.

Proof. This proof is essentially identical to the one given in [CKP15] for a similar statament. For completeness, we repeat it: Fix an index $k$. Given an adversary $A^{\prime}$ for the new obfuscator $\mathcal{O}^{\prime}$, we construct a new adversary $A^{M}$ for the $\mathcal{O}^{M}$ obfuscator as follows. The new adversary $A^{M}\left(C_{k}\right)$, on input a circuit $C_{k}$ produced by the obfuscation $\mathcal{O}^{M}$ algorithm, simulates steps 2,3, and 4 of the $\mathcal{O}^{\prime}$ algorithm by answering all queries using its oracle $M$ (whose answers will be consistent with the oracle used by $\mathcal{O}^{M}$ 
to produce $C_{k}$ ). At the end of this simulation, $A$ thus produces a circuit $C_{k}^{\prime}$ with exactly the same distribution as the output of $\mathcal{O}^{\prime}$. Adversary $A^{M}$ then runs $A^{\prime}\left(C_{k}^{\prime}\right)$ (which does not make any oracle queries) and returns the same output. It therefore follows by construction that

$$
\operatorname{Pr}\left[A^{M_{|k|}}\left(\mathcal{O}^{M_{|k|}}(k)=1\right]=\operatorname{Pr}\left[A^{\prime}\left(\mathcal{O}^{\prime}(k)\right)=1\right]\right.
$$

By the approximate VBB security property of $\mathcal{O}$ for family $F$, it follows that there exists a simulator $S$ and a negligible function $\mu$ such that

$$
\operatorname{Pr}\left[A^{M_{|k|}}\left(\mathcal{O}^{M_{|k|}}(k)=1\right]-\operatorname{Pr}\left[S^{F_{k}}(|k|)=1\right] \leq \mu(|k|)\right.
$$

which immediately implies that

$$
\operatorname{Pr}\left[A^{\prime}\left(\mathcal{O}^{\prime}(k)=1\right]-\operatorname{Pr}\left[S^{F_{k}}(|k|)=1\right] \leq \mu(|k|)\right.
$$

and concludes the proposition since $S$ is also a good simulator for $A^{\prime}$.

We conclude that $\mathcal{O}^{\prime}$ is a secure $\epsilon(n)+1 / n$-approximate VBB obfuscator for $F$ (in the plain model). This finishes the proof of Lemma 6.

Remark (extension to "sparse" high-degree zero-test polynomials) The only place in the proof where we make use of the constant-degree restriction on the zero-test queries is to argue that the number of monomials in encoded values is polynomial. It thus directly follows that the theorem extends also to high-degree polynomials as long as the legality predicate restricts these polynomials to be "sparse" in the sense that the total number of monomials over which any legal zero-test query is formed must be (a-priori) polynomially bounded. Note that it does not suffice to require that each zero-test query has a small number of monomials. Rather, we require that there exists a small set of monomials that suffices to represent all legal zero-test queries.

Remark (extension to "multi-slot" graded encodings) Our result directly extend to "multi-slot" graded encodings (as in [AB15]), which are a model of composite-order graded encodings. In this model, an encoding is a vector of elements; operations on elements are performed componentwise and finally a zero-test can be performed which determines whether the whole vector is o. Our proof directly extends also to this setting (by simply viewing each component as a separate variable). 
Remark (extension to rings) We note that our proof directly generalizes to any graded encoding scheme that operates on elements in a ring (as opposed to $\mathbb{F}_{p}$ ) as long as a) there exists an efficient method for determining the row-rank of a matrix of this ring, and) the row-rank of a matrix is polynomially bounded by the column-rank. Property a) is needed to test whether we get a linearly independent polynomial (we used Gaussian elimination for the case of $\mathbb{F}_{p}$ ), and property $b$ ) is needed to ensure that the maximum number of linearly independent polynomials is polynomially bounded by the number of monomials (for the case of $\mathbb{F}_{p}$ row-rank equals column-rank, and thus the number of linearly independent polynomials is bounded by the number of monomials).

\section{Impossibility of Subexponential VBB security}

We now consider sub-exponential VBB security and rule out constructions that use $n^{\epsilon}$-degree zero-test queries for any $0<\epsilon<1$.

Theorem 9. Assuming the existence of exponentially-secure trapdoor permutations, there exists a family of polynomial-time computable functions $F$ such that for every $0<\alpha<1$, every degree- $n^{\alpha}$ ideal graded encoding oracle $M, a$ polynomial-size 0.9-approximate VBB obfuscator for $F$ does not exist in the $M$ oracle model.

Recall that, in contrast, Barak et al $\left[\mathrm{BGK}^{+}{ }_{13}\right]$ show that for evey family $F$ of polynomial-time functions, subexponentially-secure VBB obfuscation is possible using $p(n)$-degree ideal graded encodings where $p$ is a polynomial (under appropriate cryptographic hardness assumptions).

We follow the proof Theorem 5 and prove the following lemma which combined with Theorem 3 proves the theorem.

Lemma 10. For every $\alpha<1$ and every degree- $n^{\alpha}$ ideal graded encoding oracle $M$, if a family of functions $F$ has a polynomial-size $\epsilon(n)$-approximate subexponentiallysecure VBB obfuscator in the $M$ oracle model, then there exists a subexponentialsize $(\epsilon(n)+1 / n)$-approximate subexponentially-secure $V B B$ obfuscator for $F$ in the plain model.

Proof. (sketch) The construction is identical to the one in the proof of Lemma 6 , except that we set $d=n^{\alpha}$ (instead of it being a constant), where $n=|k|$.

By the same proof, the size of the new (plain-model) obfuscator is polynomial in $\ell(n)^{n^{\alpha}}=2^{n^{\alpha}} \log \ell(n)$, where $\ell(n)$ is a bound on the number of 
encoding queries made by the original obfuscator. It follows that the size of the obfuscator is subexponential.

Approximate correctness follows in exactly the same was as in the proof of Lemma 6. Finally, subexponential VBB simulation follows in exactly the same was as Lemma 6 by appealing to subexponential VBB security of the original VBB obfuscator.

\section{References}

[AB15] Benny Applebaum and Zvika Brakerski. Obfuscating circuits via composite-order graded encoding. In Theory of Cryptography - 12th Theory of Cryptography Conference, TCC 2015, Warsaw, Poland, March 23-25, 2015, Proceedings, Part II, pages 528$556,2015$.

$\left[\mathrm{BGI}^{+}\right.$O1] Boaz Barak, Oded Goldreich, Rusell Impagliazzo, Steven Rudich, Amit Sahai, Salil Vadhan, and Ke Yang. On the (im)possibility of obfuscating programs. In Advances in Cryptology CRYPTO 2001, pages 1-18. Springer, 2001.

$\left[\mathrm{BGK}^{+}{ }^{13}\right]$ Boaz Barak, Sanjam Garg, Yael Tauman Kalai, Omer Paneth, and Amit Sahai. Protecting obfuscation against algebraic attacks. Cryptology ePrint Archive, Report 2013/631, 2013.

$\left[\mathrm{BGK}^{+}{ }_{14}\right]$ Boaz Barak, Sanjam Garg, Yael Tauman Kalai, Omer Paneth, , and Amit Sahai. Protecting obfuscation against algebraic attacks. In EUROCRYPT'14, 2014.

[BP13] Nir Bitansky and Omer Paneth. On the impossibility of approximate obfuscation and applications to resettable cryptography. In $S T O C^{\prime} 13,2013$.

[BR93] Mihir Bellare and Phillip Rogaway. Random oracles are practical: A paradigm for designing efficient protocols. In CCS '93, Proceedings of the 1st ACM Conference on Computer and Communications Security, Fairfax, Virginia, USA, November 3-5, 1993., pages 62-73, 1993 .

[BR14a] Zvika Brakerski and Guy N. Rothblum. Virtual black-box obfuscation for all circuits via generic graded encoding. In TCC, pages 1-25, 2014. 
[BR14b] Zvika Brakerski and Guy N. Rothblum. Virtual black-box obfuscation for all circuits via generic graded encoding. In TCC'14, 2014.

[BSo3] Dan Boneh and Alice Silverberg. Applications of multilinear forms to cryptography. Contemporary Mathematics, 324(1):7190, 2003 .

[Can97] Ran Canetti. Towards realizing random oracles: Hash functions that hide all partial information. In Advances in Cryptology CRYPTO 1997, pages 455-469, 1997.

[CKP15] Ran Canetti, Yael Tauman Kalai, and Omer Paneth. On obfuscation with random oracles. In TCC'15, 2015.

[CV13] Ran Canetti and Vinod Vaikuntanathan. Obfuscating branching programs using black-box pseudo-free groups. Cryptology ePrint Archive, Report 2013/500, 2013. https:/ / eprint.iacr.org/2013/500.

[GGH13a] Sanjam Garg, Craig Gentry, and Shai Halevi. Candidate multilinear maps from ideal lattices. In Advances in CryptologyEUROCRYPT 2013, pages 1-17. Springer, 2013.

$\left[\mathrm{GGH}^{+}{ }^{13} \mathrm{~b}\right]$ Sanjam Garg, Craig Gentry, Shai Halevi, Mariana Raykova, Amit Sahai, and Brent Waters. Candidate indistinguishability obfuscation and functional encryption for all circuits. Proc. of FOCS 2013, 2013.

[Hadoo] Satoshi Hada. Zero-knowledge and code obfuscation. In Advances in Cryptology-ASIACRYPT 2000, pages 443-457. Springer, 2000.

[LPSo4] Ben Lynn, Manoj Prabhakaran, and Amit Sahai. Positive results and techniques for obfuscation. In Advances in Cryptology - EUROCRYPT 2004, International Conference on the Theory and Applications of Cryptographic Techniques, Interlaken, Switzerland, May 2-6, 2004, Proceedings, pages 20-39, 2004.

[PST13] Rafael Pass, Karn Seth, and Sidharth Telang. Indistinguishability obfuscation from semantically-secure multilinear encodings. Cryptology ePrint Archive, Report 2013/781, 2013. 
[Rot13] Ron D Rothblum. On the circular security of bit-encryption. In Theory of Cryptography, pages 579-598. Springer, 2013.

[Sho97] Victor Shoup. Lower bounds for discrete logarithms and related problems. In Advances in Cryptology - EUROCRYPT '97, International Conference on the Theory and Application of Cryptographic Techniques, Konstanz, Germany, May 11-15, 1997, Proceeding, pages 256-266, 1997. 